\title{
High Resolution Imaging Analysis of Core/Shell CdSe/ZnS Quantum Dots (QDs) and Graphene QDs using Cs-corrected HR-STEM/TEM
}

\author{
Huiyoun Shin ${ }^{1}$, Youngil. Jang ${ }^{1}$, Dongseon. Jang ${ }^{2}$, Myungju. Cho ${ }^{1}$, and Kyuho Park ${ }^{1}$ \\ ${ }^{1 .}$ Materials Characterization Team, Materials \& Components Laboratory, LG Electronics Advanced \\ Research Institute, 16 Woomyeon-dong, Seocho-gu, Seoul 137-724, South Korea \\ 2. New Device Team, Future Device R\&D Department, LG Electronics Advanced Research Institute, 16 \\ Woomyeon-dong, Seocho-gu, Seoul 137-724, South Korea.
}

Nano-crystal quantum dots (QDs) are ideal candidates for light-emission applications due to their high quantum efficiency, narrow-band and particle-size-tunable photoluminescence. In particular, their small size results in the quantum confinement of the semiconductor nano-crystals, which widens their energy gaps. Their size-tunable optical properties are of major interest for lighting and solar cell applications and for replacement of fluorescent dyes in biological imaging $[1,2]$.

QDs with emissions in the spectral range from 525 to $535 \mathrm{~nm}$ are of special interest for application in green LEDs and white-light generation. Of all QDs in the spectral range, CdSe/ZnS core-shell structured nano-crystals are among the promising candidates [3]. On the other hand, graphene quantum dots (GQDs) have attracted great attention for their unique spin, electronic and optical properties, so several approaches have been developed to fabricate GQDs [4,5].

Transmission electron microscope (TEM) equipment has opened a wide range of opportunities for structural characterization of materials, due to its excellent spatial resolution and various capabilities. Nonetheless, sample preparation for observing the TEM has become a limiting factor in the many fields. TEM imaging relies upon difference between the electron densities of the sample and the surrounding substrate/matrix. For successful imaging of nano-particles, it depends on the contrast of the QDs sample relative to the supporting film. In this work, samples for observing QDs (CdSe/ZnS and GOD) were prepared by drying each QDs solution on copper grids that were coated with a graphene monolayer and a SiO membrane. All TEM and STEM analyses were performed on an FEI Titan G2 (80-300kV) equipped with a probe Cs corrector.

Nano-structure of the core/shell CdSe/ZnS QDs was observed from HRTEM and HRSTEM modes in figure 1. The QDs assembled on the $\mathrm{Cu}$ grid with the graphene monolayer (thickness $=0.3 \mathrm{~nm}$ ). Using a ultra-thin graphene membrane, we could obtain high quality HRTEM and HRSTEM HAADF images of the QDs. Figure 1(a) and (c) show that the size distribution of green colored QDs is concentrated in the range 5-6 $\mathrm{nm}$. It was originally hoped that this technique would reveal the $\mathrm{ZnS}$ shell via $\mathrm{Z}$-contrast, but the shell was not visualized clearly using STEM HAADF.

Observation of the GODs using TEM was surprisingly successful in figure 2. For TEM analysis of GODs, the $\mathrm{Cu}$ grid coated with a carbon membrane could not be used because the GODs had the same element component as the carbon membrane. Therefore, we used the $\mathrm{Cu}$ grid coated with a $\mathrm{SiO}$ membrane (thickness $=30 \mathrm{~nm}$ ) to assemble GODs. We could distinguish the GQDs from the $\mathrm{SiO}$ background. From the figure 2, the sizes of GODs were evaluated to be about $8 \mathrm{~nm}$. The high crystallinity of the GQDs was identified from the lattice fringes of HRTEM. 
References:

[1] G. Jacob, L.C. Barbosa and C.L. Cesar, in Proceeding of the SPIE 5734 (2005), 124.

[2] B. Ballou, B.C. Lagerholm, L.A. Ernst and A.S. Waggoner, Bioconjugate Chem. 15 (2004), 79.

[3] Weiling Luan, Hongwei Yang and Shan-Tung Tu, Nanoscale Res Lett. 3 (2008), 134-135.

[4] Ritter, and K.A. Lyding, Nat. Mater. 8 (2009), 235-236.

[5] Pan, D., Zhang, J. Li, Z. and Wu, M., Adv. Mater. 22 (2010), 734.

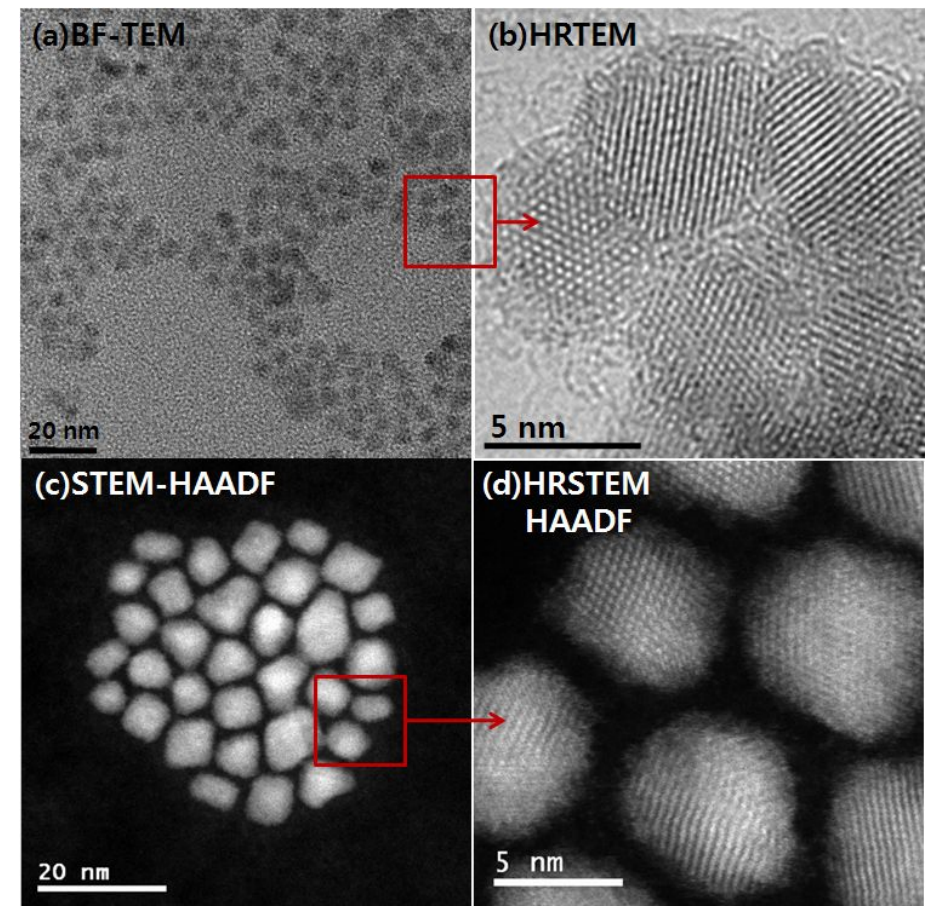

Figure 1. High resolution images of core/shell $\mathrm{CdSe} / \mathrm{ZnS}$ QDs. The QDs assembled on the $\mathrm{Cu}$ grid coated with a graphene monolayer (thickness $=0.3 \mathrm{~nm}$ ).

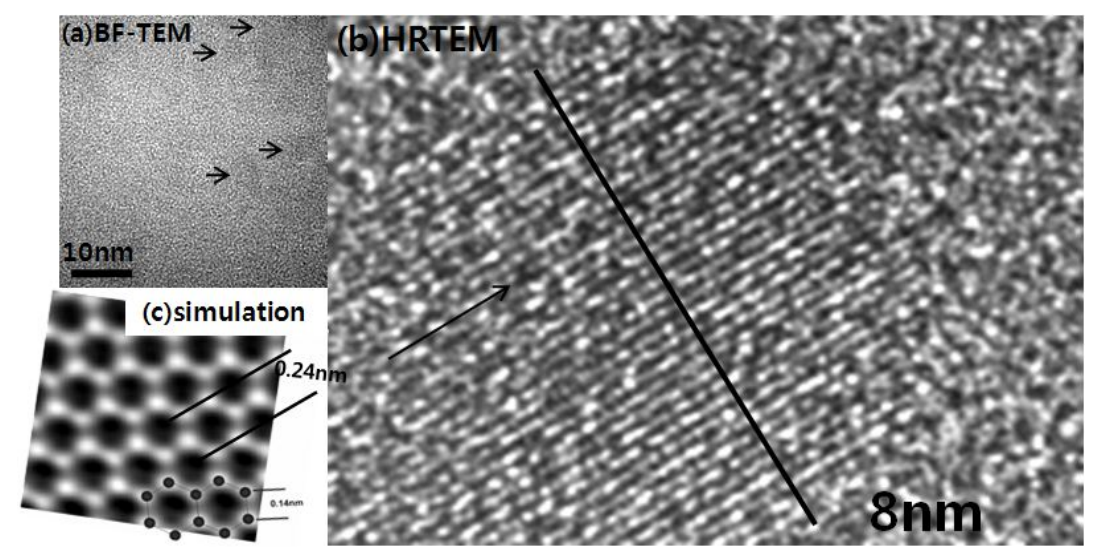

Figure 2. Observed(a,b) and simulated(c) images of graphene QDs. The GQDs assembled on the $\mathrm{Cu}$ grid coated with a $\mathrm{SiO}$ membrane, and the GQDs were distinguished from the $\mathrm{SiO}$ background. 